\title{
Molecular diffusion and dc conductivity perfectly correlated with molecular rotational dynamics in a plastic crystalline electrolyte
}

\author{
M. Zachariah, M. Romanini, P. Tripathi, J. LI. Tamarit and R. Macovez \\ We probe the ion conduction and the molecular dynamics in a pure and lithium-salt doped dinitrile molecular plastic \\ crystal. While the diffusion of the $\mathrm{Li}^{+}$ions is decoupled from the molecular reorientational dynamics, in the undoped \\ plastic crystal the temperature dependence of the mobility of dinitrile ions and thus of the conductivity is virtually identical \\ to that of on-site molecular rotations. The undoped material is found to obey the Walden and Stokes-Einstein rules typical \\ of ideal liquid electrolytes, implying that an effective viscosity against diffusion can be defined even for a plastic crystalline \\ phase. These surprising results, never reported before in a translationally ordered solid, indicate that in this dinitrile plastic \\ crystalline material the time scale of translational diffusion is perfectly correlated with that of the purely reorientational \\ on-site dynamics.
}

\section{Introduction}

Diffusion processes are responsible for many properties such as viscosity, plasticity, or heat and charge transport. In liquids, the diffusion of the constituent molecules accounts for the fluidity and viscosity of the medium, while diffusion of dissolved charged species results in electrical conduction. These two diffusion processes are mutually coupled because the ionic motion is limited by the viscosity; as a result, solutions of large, weakly coordinating ions in solvents with nonspecific ion-solvent interactions obey the so-called StokesEinstein relation, ${ }^{1}$ according to which $\mathrm{k}_{\mathrm{B}} T \eta / D \simeq$ const, where $\mathrm{k}_{\mathrm{B}} T$ is the thermal energy, $D$ is the diffusivity of the ions and $\eta$ is the viscosity. Because the ionic diffusivity is proportional to the electrical conductivity $\sigma_{\mathrm{dc}}$, the StokesEinstein relation implies that the plot of $\log \left(\sigma_{\mathrm{dc}}\right)$ versus $\log (\eta)$ has a slope equal to one, an empirical relation known as Walden rule and verified in many liquid electrolytes. ${ }^{2}$

Also in the solid state the diffusion of ions gives rise to charge transport, but since usually the concepts of viscosity and of molecular diffusion are not useful to describe crystalline solids, no equivalent of the Stokes-Einstein or Walden relations exists. In condensed phases other than ideal ionic solutions, the validity of the Walden rule was only reported in some disordered systems such as glass-forming supercooled liquids or polymers. ${ }^{3}$ There is, however, a class of ordered solids in which molecular dynamics are extremely important, namely plastic crystals. Plastic crystals are molecular solids which display long-range translational order and dynamic orientational disorder. Their constituent molecules undergo free-rotor or large-angle rotational motions about their (average) centres of mass, which occupy fixed lattice positions. ${ }^{4,5}$ The "plasticity" of these orientationally disordered phases arises from the presence of lattice defects near which the molecules exhibit also diffusion dynamics.
It has been suggested that ion transport in plastic crystals is enhanced by the rotational dynamics, and several efforts have been directed in the past few years to investigate a possible connection between molecular reorientations and ion diffusion. ${ }^{6}$ For example, in the plastic-crystal phase of phosphonium hexaphosphate the ionic conduction is enhanced by the rotational motion of the phosphonium ions. ${ }^{7}$ In some organic plastic-crystalline electrolytes it has been even proposed that the ionic motion may be boosted via a so-called "paddling wheel" mechanism, which should result in a perfect correlation between ion diffusion and molecular reorientational dynamics. $^{8-11}$ However, so far such a correlation has been elusive. Probing and understanding the connection between ion diffusion and molecular dynamics in plastic crystals is important from a fundamental perspective but also for application purposes. In fact, plastic-crystalline electrolytes are ideal for portable batteries because their mechanical flexibility reduces the problem of poor electrical contact with the electrodes caused by thermal volume changes of solid electrolytes, while eliminating the leakage problems associated with liquid electrolytes. ${ }^{11,12}$

We study here a plastic crystalline electrolyte based on the small, high-symmetry succinonitrile molecule $\left(\mathrm{SN}, \mathrm{C}_{2} \mathrm{H}_{4}(\mathrm{CN})_{2}\right)$. Near room temperature, $\mathrm{SN}$ is well-known to exhibit plasticcrystalline characteristics combined with unusually high ion mobility, a feature that has been exploited in the last decade to achieve high-conductivity solid electrolytes. ${ }^{13-17}$ Between 331 and $233 \mathrm{~K}$, pure SN forms a plastic crystal phase with body-centred cubic ( $b c c$ ) lattice in which the molecules, which exist in two conformations, ${ }^{18,19}$ have their central $\mathrm{C}-\mathrm{C}$ bond parallel to one of the diagonals of the cubic cell. The disorder in this plastic-crystalline phase is associated with isomeric fluctuations and with large-angle molecular rotations in which the $\mathrm{C}-\mathrm{C}$ bond orientation jumps from one diagonal position of the cubic cell to another. ${ }^{13,18-20}$ Below $233 \mathrm{~K}$, pure SN displays a perfectly crystalline monoclinic phase without molecular 
motions nor isomerism. ${ }^{20,21}$ In spite of the widespread belief that the orientational dynamic processes in plastic SN are beneficial to its ionic mobility, ${ }^{20,22}$ no clear correlation has been found between both quantities. In fact, while earlier studies on Li-doped SN did not address the issue of correlation between conduction and molecular dynamics, ${ }^{23,24}$ a more recent study on a nearly equimolar mixture of succinonitrile with the similar glutaronitrile compound $\left(\mathrm{GN}, \mathrm{C}_{3} \mathrm{H}_{6}(\mathrm{CN})_{2}\right)$ has shown that the $\mathrm{dc}$ conductivity and the molecular reorientational dynamics have actually distinct the temperature dependence and are thus effectively decoupled. ${ }^{25}$ If such decoupling took place also in pure $\mathrm{SN}$, it would indicate that its relatively high ionic mobility is merely an effect of the low density of the plastic phase ${ }^{26}$ rather than of the rotational dynamics, as generally supposed. ${ }^{8-11}$ To elucidate these matters, we have measured by means of dielectric spectroscopy the dc conductivity and molecular dynamics of pure and lithium-salt doped binary GN-SN mixtures at low glutaronitrile weight percentage (15\%). Besides yielding relatively good miscibility even in the solid state, ${ }^{25,27}$ the addition of GN in sufficient amount effectively suppresses the transition to the fully ordered monoclinic phase, thus enlarging the temperature range of the plastic-crystal phase. ${ }^{25}$ The $15 \% \mathrm{GN}-85 \% \mathrm{SN}$ mixture is the binary stoichiometry closest to pure SN that yields a plastic phase stable in a large temperature interval (see Fig. 1 of Ref. 25 or Fig. 2 of Ref. 27).

\section{Results and discussion}

Fig. 1 shows the dielectric spectra of the $15 \% \mathrm{GN}-85 \% \mathrm{SN}$ mixture acquired between 140 and $250 \mathrm{~K}$. The loss spectra (imaginary part of the permittivity $\varepsilon$, Fig. 1a) exhibit a prominent peak that shifts to lower frequency as the temperature is decreased. This loss feature is observed at roughly the same frequencies as the dipolar relaxation of pure SN above $233 \mathrm{~K}$ and of the GN-SN binary system at high GN content. ${ }^{25,27}$ Such dielectric feature, referred to as $\alpha$ relaxation, is the spectral signature of the collective reorientational motions of the dinitrile molecules. Upon decreasing the temperature the dynamics slows down, reaching a frequency below $10^{-1} \mathrm{~Hz}$ at $145 \mathrm{~K}$, the reported glass transition temperature. $^{25}$ The dielectric loss mainly stems from the purely rotational motion of molecules occupying a fixed position in the bcc lattice, as only a minor fraction of the molecules exhibits rototranslational diffusion near lattice defects. $^{7,11,28,29}$

The ac conductivity spectra (Fig. $1 b$ ) exhibit a horizontal plateau at intermediate or low frequency, which corresponds to the dc conductivity regime. The decrease of conductivity (spectral bending) at the lowest frequencies, visible especially at high temperature, is due to the accumulation of ions at the electrode-sample interface. The value of the dc conductivity of the GN-SN mixture is remarkably high considering that both dinitrile molecules are neutral closed-shell molecules, so that no electronic or ionic conduction is expected; such a high value is indicative of a very high mobility of ionic impurities in the system. The conductivity varies by six decades in an interval of $\sim 80 \mathrm{~K}$ between 240 and $160 \mathrm{~K}$. In the same range also the peak position in the loss spectra is observed to shift by approximately six decades, while it shifts by more than nine decades over the full measurable temperature interval down to $145 \mathrm{~K}$.

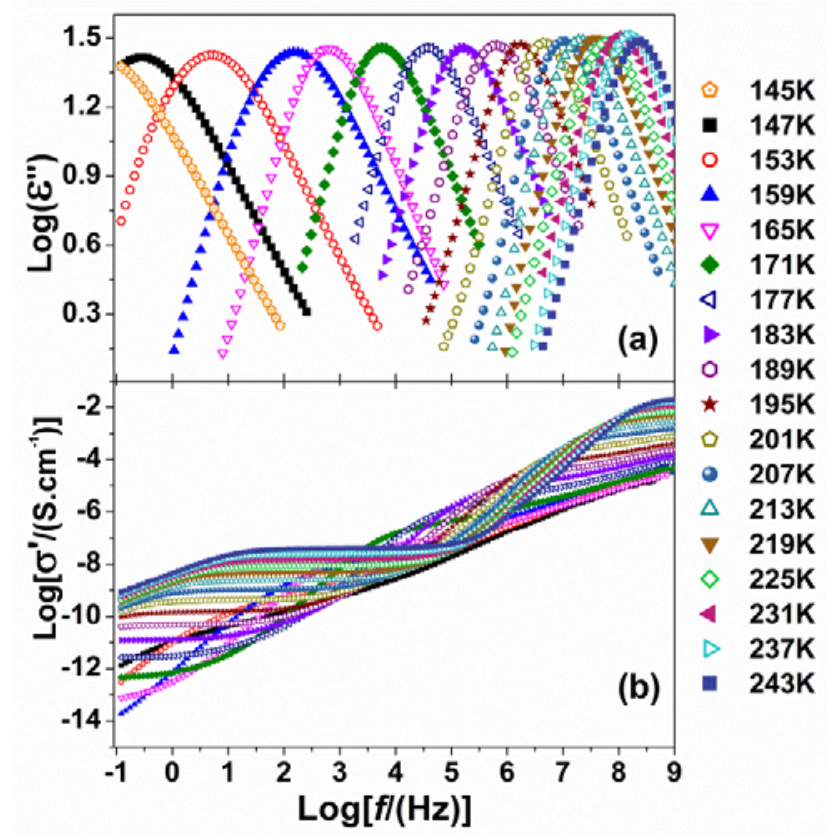

Figure 1. Dielectric loss $\varepsilon^{\prime \prime}(f)(a)$ and ac conductivity $\sigma^{\prime}(f)(b)$ spectra of the plastic phase of the $15 \% \mathrm{GN}-85 \% \mathrm{SN}$ mixture, at the indicated temperatures.

From Fig. 1 one may obtain, for each temperature, the value of the dc conductivity $\sigma_{\mathrm{dc}}$ and the characteristic frequency $f_{\max }$ at which the dielectric loss is maximum. To obtain $f_{\max }$ the loss spectra were fitted assuming a Havriliak-Negami profile ${ }^{30}$ of the $\alpha$ relaxation. The value of $\sigma_{\mathrm{dc}}$ was taken to be value of the ac conductivity spectra in the middle of the horizontal plateau, that is, at the frequency where the imaginary part of $1 / \varepsilon$ (modulus) was maximum.

Fig. 2 shows the plots of $f_{\max }$ and of $\sigma_{\mathrm{dc}}$ of the undoped GN-SN mixture versus inverse temperature, together with the analogous values obtained after mixing it with $\mathrm{LiBF}_{4}$ so as to achieve a ternary mixture with $2 \% \mathrm{LiBF}_{4}, 14 \% \mathrm{GN}$ and $84 \% \mathrm{SN}$ in weight. Upon doping the mixture with $\mathrm{LiBF}_{4}$, the dc conductivity increased due to the higher ion density. ${ }^{13-15}$ The characteristic frequency of the relaxation is lower in the doped mixture (especially at low $T$ ) due to the stronger interactions induced by the presence of $\mathrm{Li}^{+}$and $\mathrm{BF}_{4}{ }^{-}$ions. ${ }^{31}$ Also the $\mathrm{dc}$ conductivity values are quite different: not only is $\sigma_{\mathrm{dc}}$ higher when $\mathrm{Li}^{+}$ions are present, but it also spans a larger number of decades between 240 and $160 \mathrm{~K}$ (instead, the relaxation frequency spans a smaller number of decades, in a given temperature interval, when $\mathrm{Li}^{+}$ions are present). 

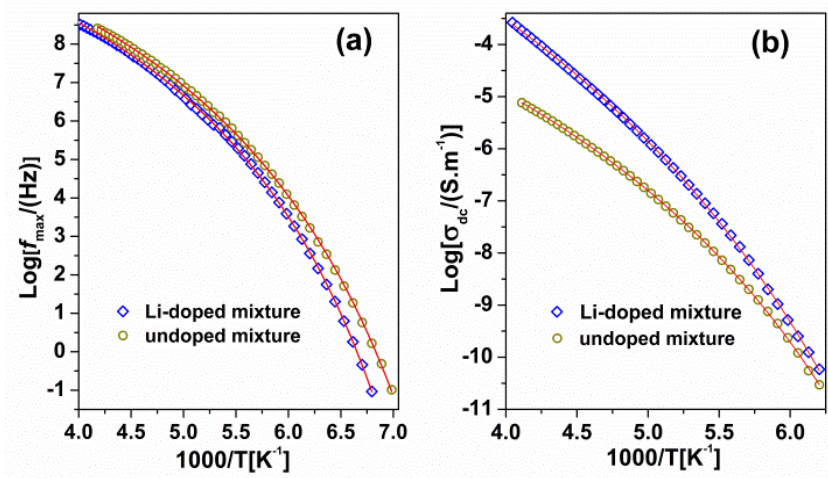

Figure 2. Semilogarithmic plot of $f_{\max }(a)$ and of $\sigma_{\mathrm{dc}}(b)$ vs 1000/T, for the pure and $\mathrm{LiBF}_{4}$ doped GN-SN mixture. Continuous lines are fits with eq.s (1) and (2), respectively.

As it is rather common in glass forming materials and plastic crystals, the relaxation frequency exhibits a non-simplyactivated behaviour (i.e., it does not scale linearly with the inverse temperature). ${ }^{32,33}$ A widespread approach ${ }^{32}$ to model the non-linear inverse-temperature dependence of the $\alpha$ relaxation is the so-called Vogel-Fulcher-Tammann (VFT) equation, which is given by: ${ }^{30}$

(1) $f_{\max }=f_{0} \exp \left[-s T_{\mathrm{VF}} /\left(T-T_{\mathrm{VF}}\right)\right]$.

Here the prefactor $f_{0}$, the strength parameter $s$ and the socalled Vogel-Fulcher temperature $T_{\mathrm{VF}}$ are phenomenological parameters. Similarly, the dc conductivity can be described as:

(2) $\sigma_{d c}=c_{0} T \exp \left[-s^{\prime} T_{V F}^{\prime} /\left(T-T_{V F}^{\prime}\right)\right]$

As visible in Fig. 2, eq.s (1) and (2) give quite accurate fits. The fit parameters are summarized in Table 1.

Table 1. Fit parameters obtained for the indicated samples using the Vogel-FulcherTammann eq.s (1) and (2).

\begin{tabular}{|c|c|c|c|}
\hline$\% G N-\% S N-\% s a l t$ & prefactor & $s$ & $T_{\mathrm{VF}}(\mathrm{K})$ \\
\hline$f_{\max }(15-85-0)$ & $\begin{array}{c}f_{0}=1.48 \pm 0.11 \\
10^{12} \mathrm{~Hz}\end{array}$ & $9.7 \pm 0.1$ & $105.6 \pm 0.3$ \\
\hline$\sigma_{\mathrm{dc}} / T(15-85-0)$ & $\begin{array}{c}c_{0}=1144 \pm 50 \\
\mathrm{~S} \mathrm{~cm}^{-1} \mathrm{~K}^{-1}\end{array}$ & $10.0 \pm 0.1$ & $107.3 \pm 0.3$ \\
\hline$f_{\max }(14-84-2)$ & $\begin{array}{c}f_{0}=0.86 \pm 0.06 \\
10^{12} \mathrm{~Hz}\end{array}$ & $10.8 \pm 0.1$ & $111.0 \pm 0.3$ \\
\hline$\sigma_{\mathrm{dc}} / T(14-84-2)$ & $\begin{array}{c}\mathrm{C}_{0}=3.0 \pm 0.4 \\
\mathrm{~S} \mathrm{~cm} \mathrm{~K}^{-1}\end{array}$ & $15.4 \pm 0.3$ & $98.9 \pm 0.6$ \\
\hline
\end{tabular}

The analytical form of the prefactors in the VFT eq.s (1) and (2) is to some extent arbitrary, because the temperature dependence of $\sigma_{\mathrm{dc}}$ and $f_{\max }$ is largely dominated by the exponential term, so that one may assume any slowly varying function of $T$ in the prefactor. An increase of the conductivity prefactor with temperature can be rationalized in light of recent studies of ion conduction in polar electrolytes ${ }^{34,35}$ (see ESI file).

We can now discuss the correlation between ionic conductivity and orientational dynamics in the pure $15 \% \mathrm{GN}-85 \% \mathrm{SN}$ mixture. The first evidence that these two quantities are correlated (in the absence of lithium ions) is the fact that both $\sigma_{\mathrm{dc}}$ and $f_{\max }$ vary by the same number of decades in the same temperature interval. Also, the Vogel-Fulcher temperature and the strength parameter are almost identical for both quantities (see Table 1). To further corroborate the connection between the reorientational dynamics and the conductivity, Fig. $3 a$ shows the plot of $\log \left(\sigma_{\mathrm{dc}}\right)$ versus $\log \left(f_{\text {max }}\right)$. The correlation between the two quantities is perfect, with a slope virtually equal to one. This entails that the motion of charge carriers in plastic SN is perfectly correlated with the reorientational motion of the dinitrile molecules. The linear correlation does not hold for the lithium-doped sample (especially at high $T$, see Fig. 3a), which indicates that the connection between molecular reorientation and charge carrier diffusion does not hold for the small $\mathrm{Li}^{+}$ions.

The logarithmic plot of Fig. $3 a$ is analogous to the Walden plot for liquid electrolytes. In a liquid, $f_{\max }$ corresponds to the characteristic frequency at which (rototranslational) molecular diffusion takes place, and it is inversely proportional to the viscosity $\eta$ of the medium. ${ }^{30}$ The connection between ion diffusion and molecular reorientations in our mixed plastic crystal can be best corroborated by looking at the behaviour of the quantity $\sigma_{\mathrm{dc}} /\left(\mathrm{k}_{\mathrm{B}} T \cdot f_{\mathrm{max}}\right)$, defined in analogy to the StokesEinstein relation, which states that the product of the limiting molar conductivity and the solvent's viscosity $\eta$ (or equivalently $1 / f_{\max }$ ) is constant for diluted electrolyte solutions. Fig. $3 b$ shows that this ratio is basically constant for the undoped mixture in the whole accessible temperature range.

The observation of a one-to-one correspondence between the conductivity and the relaxation frequency in a linear plot provides a definitive proof of the perfect correlation between molecular reorientations and ionic conductivity in this plasticcrystalline electrolyte. The validity of the Walden rule can be thought of as a consequence of the relation $\sigma_{\mathrm{dc}} /\left(\mathrm{k}_{\mathrm{B}} T \cdot f_{\max }\right)=$ const : as both $\sigma_{\mathrm{dc}} / T$ and $f_{\max }$ depend exponentially on temperature, $\log \left(\sigma_{\mathrm{dc}} / T\right)$ and $\log \left(f_{\text {max }}\right)$ are almost perfectly correlated. The analogy with the Stokes-Einstein relation rationalizes our choice of prefactor in eq. (2).
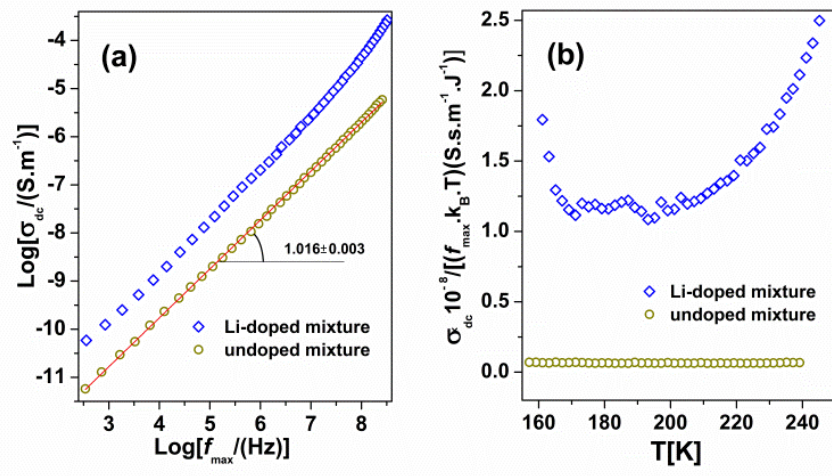

Figure 3. Walden plot $(a)$ and plot of $\sigma_{\mathrm{dc}} /\left(\mathrm{k}_{\mathrm{B}} T \cdot f_{\mathrm{max}}\right)$ versus temperature $(b)$ for the pure and $\mathrm{LiBF}_{4}$-doped GN-SN mixture (data of Fig. 2). The continuous line is a linear fit.

The validity in a plastic crystal of relations equivalent to the Stokes-Einstein and Walden rules is surprising, given that the concept of viscosity against ion diffusion is not expected to apply to a crystalline solid. To the best of our knowledge, this is the only translationally ordered solid phase where such a connection has ever been reported. The fulfilment of relations 
typical of liquid electrolytes confirms and substantiates an early, suggestive report of "liquid-like" diffusion properties of the plastic SN electrolyte. ${ }^{15}$

As pointed out earlier, the value of $\sigma_{\mathrm{dc}}$ of plastic $\mathrm{SN}$ and dinitrile mixtures is surprisingly high. A likely explanation for the relatively high $\sigma_{\mathrm{dc}}$ values is that they are due to a minority of dinitrile molecules present as molecular ions. Given that in plastic crystals a fraction of the molecules undergoes diffusive motions between lattice defects, ${ }^{7,11,15,28,29}$ charge transport can result from diffusion of ionized molecules. The validity of the Walden and Stokes-Einstein relations for the GN-SN mixture would then imply a one-to-one correlation between the molecular orientational motion and the diffusion of molecular ions. The comparison with the lithium-doped mixture validates this idea. When the dominant charge carriers are $\mathrm{Li}^{+}$ions, the temperature dependence of the conductivity is no longer perfectly correlated with that of the molecular rotational dynamics, as seen in Fig.s 2 and $3 a$, hence the motion of the $\mathrm{Li}^{+}$ions is decoupled ${ }^{6}$ from (occurs on a faster timescale than) that of the organic matrix. This rules out a paddling-wheel mechanism ${ }^{8-11}$ for $\mathrm{Li}^{+}$ion conduction, and indicates that electrical conduction in the undoped mixture is due to molecular rather than atomic ions.

Because translational and rotational degrees of freedom are generally considered to be independent, a perfect connection between the translational diffusion and the on-site orientational motion is at first quite surprising. Nonetheless, recent studies on compounds such as ethanol and glutaronitrile, which depending on the thermal treatment display (at the same temperature) either a plastic crystalline state or a supercooled liquid phase, have shown ${ }^{27,36}$ that the characteristic diffusional frequency in the supercooled liquid is at all temperatures very close to that of reorientational motions in the plastic crystal. Our findings are therefore a further confirmation of the idea that the dynamics of glassforming systems is fundamentally determined by rotational motions; ${ }^{27,36-40}$ while the connection between translational and rotational timescales was until now only based on the observation of similar but not identical temperature dependence and frequency ranges in different phases, we report here a perfect correlation of translational and rotational degrees of freedom in the same phase. The correspondence of $\sigma_{\mathrm{dc}}$ and $f_{\max }$ originates because both quantities are fundamentally linked to the diffusion of molecular species between lattice defects, which can be thought to be limited by an effective "diffusional" viscosity $\eta_{\text {diff. }}$.

It is quite unexpected that a viscosity can be defined for a translationally ordered solid, and even more so that it is perfectly correlated with the characteristic time for on-site molecular rotations. To the best of our knowledge, no other plastic crystal is known to exhibit a similar behaviour. The lack of a similar correlation in the quasi-equimolar GN-SN mixture reported in Ref. 25 might arise from the non-perfect miscibility of the two dinitrile molecules, leading to phase segregation and thus to a conductivity limited by phase-boundary effects. This view is supported by the observation in the equimolar mixture $^{25}$ of a conductivity-related dielectric loss likely associated with the sample's heterogeneities, ${ }^{41,42}$ which we did not observe in our SN-rich sample.

\section{Experimental}

Succinonitrile, glutaronitrile and $\mathrm{LiBF}_{4}$ (Sigma Aldrich) were mixed in the correct proportions to obtain the desired stoichiometry. The mixture without salt was obtained by dissolving the succinonitrile powder in glutaronitrile, which is liquid at ambient conditions. The lithium-containing mixture was obtained by melting the glutaronitrile-succinonitrile mixture at $330 \mathrm{~K}$ and then adding the correct proportion of the $\mathrm{LiBF}_{4}$ salt, preheated to $400 \mathrm{~K}$ under nitrogen atmosphere to avoid the possible presence of water. Sonication at $330 \mathrm{~K}$ (above the melting point of the mixtures) was used to favour a homogeneous mixing in both cases.

Dielectric spectroscopy yields the complex permittivity of a sample as a function of frequency. In dielectric measurements, an ac electric field is applied to a parallel-plate capacitor formed by a homogeneous sample sandwiched between two metal disks. Using the known surface area and thickness of the dielectric sample, the complex permittivity is directly extracted from the complex impedance of the capacitor cell, which is measured with an impedance analyzer.

For the dielectric measurements, the sample was inserted in liquid form inside a home-made stainless steel parallel-plate capacitor with the two plates separated by needle-like cylindrical silica spacers of $50 \mu \mathrm{m}$ diameter. The capacitor was then loaded in a nitrogen-gas flow cryostat for temperature control. Dielectric spectra were acquired in the frequency $(f)$ range from $10^{-2}$ to $10^{9} \mathrm{~Hz}$. Isothermal frequency scans were acquired cooling the sample from the liquid phase to ensure the maximum homogeneity. For measurements between $10^{-2}$ to $5 \cdot 10^{6} \mathrm{~Hz}$ a Novocontrol Alpha analyzer was employed, while for measurements between $10^{6}$ and $10^{9} \mathrm{~Hz}$, a HP4291 impedance analyzer was employed in reflectometry geometry, with the sample capacitor mounted at the end of a coaxial cable. Dielectric spectroscopy allows probing in the same experiment both the dc conductivity and the molecular dipolar dynamics. The dielectric spectra may be displayed for example as real permittivity $\varepsilon^{\prime}(f)$, dielectric loss $\varepsilon^{\prime \prime}(f)$, and ac conductivity $\sigma^{\prime}(f)$. The latter two representations are related by $\sigma^{\prime}(f)=2 \pi f \varepsilon_{0} \varepsilon^{\prime \prime}(f)$.

\section{Conclusions}

We reported the first and so-far only known example of molecular plastic crystal exhibiting a perfect agreement between the temperature dependence of the long-range ion drift and that of the collective reorientational dynamics. This correlation is absent in the lithium-doped material, which indicates that it is only valid when charge transport is dominated by diffusion of molecular ions, while the motion of the smaller $\mathrm{Li}^{+}$ions is decoupled from the rotational dynamics. These results entail that an effective viscosity against molecular diffusion can be defined even for a plastic crystal, 
and provide the first evidence that translational and orientational timescales match perfectly in the same translationally ordered phase. Our findings provide valuable knowledge of the conduction mechanism in commercially relevant electrolytes such as neutral and ionic plastic crystals, and may allow a fuller understanding of the correlation between ion diffusion and molecular dynamics in orientationally disordered phases.

\section{Acknowledgements}

This work has been partially supported by the Spanish Ministry of Science and Innovation through project FIS2011-24439 and by the Generalitat de Catalunya under project 2014 SGR-581.

\section{Notes and references}

1 J. Barthel, H. Krienke and W. Kunz, Physical Chemisty of Electrolyte Solutions (Steinkopff-Verlag, Darmstadt, 1998)

2 P.Z. Walden, Phys. Chem., 1906, 55, 207

3 C. Do, P. Lunkenheimer, D. Diddens, M. Götz, M. Weiß, A. Loidl, X.-G. Sun, J. Allgaier and M. Ohl, Phys. Rev. Lett., 2013, 111, 018301

4 R. Macovez, A. Goldoni, L. Petaccia, P.A. Brühwiler and P. Rudolf, Phys. Rev. Lett., 2008, 101, 236403

5 M. Romanini, Ph. Negrier, J. LI. Tamarit, S. Capaccioli, M. Barrio, L. C. Pardo and D. Mondieig, Phys. Rev. B, 2012, 85, 134201

6 Z. Wojnarowska, Y. Wang, J. Pionteck, K. Grzybowska, A.P. Sokolov and M. Paluch, Phys. Rev. Lett., 2013, 111, 225703

7 L. Jin, K.M. Nairn, C.M. Forsyth, A.J. Seeber, D.R. MacFarlane, P.C. Howlett, M. Forsyth and J.M. Pringle, J. Am. Chem. Soc., 2012, 134, 9688

8 D.R MacFarlane, P. Meakin, N. Amini and M. Forsyth, J. Phys.: Condens. Matter, 2001, 13, 8257

9 S. Murugesan, O.A. Quintero, B.P. Chou, P. Xiao, K. Park, J.W. Hall, R.A. Jones, G. Henkelman, J.B. Goodenough and K.J. Stevenson, J. Mater. Chem. A, 2014, 2, 2194

10 M. Jansen, Angew. Chem. Int. Ed., 1991, 30, 1547

11 D.R. MacFarlane and M. Forsyth, Adv. Mater., 2001, 13, 957

12 J. Sunarso, Y. Shekibi, J. Efthimiadis, L. Jin, J. Pringle, A Hollenkamp, D.R. MacFarlane, M. Forsyth and P. Howlett, J. Solid State Electrochem., 2012, 16, 1841

13 S. Long, D.R. MacFarlane and M. Forsyth, Solid State lonics, 2003, 161, 105

14 P.-J. Alarco, Y. Abu-Lebdeh, A. Abouimrane, and M. Armand, Nat. Mater., 2004, 3, 476

15 Q. Dai, D.R. MacFarlane, P.C. Howlett and M. Forsyth, Angew. Chem. Int. Ed., 2005, 44, 313

16 D. Hwang, D.Y. Kim, S.M. Jo, V. Armel, D.R. MacFarlane, D. Kim and S.-Y. Jang, Sci. Rep., 2013, 3, 3520

17 K.-H. Choi, S.-J. Cho, S.-H. Kim, Y.H. Kwon, J.Y. Kim and S.-Y. Lee, Adv. Funct. Mater., 2014, 24, 172

18 G. Cardini, R. Righini and S. Califano, J. Chem. Phys., 1991, 95, 679

19 P. Derollez, J. Lefebvre, M. Descamps, W. Press and H. Fontaine, J. Phys. Condens. Matter, 1990, 2, 6893

20 H.M. Hawthorne and J.N. Sherwood, Trans. Faraday Soc., 1970, 66, 1792

21 A. Masui, S. Yoshioka, and S. Kinoshita, Chem. Phys. Lett. 2001, 341, 299

22 M. Patel, P.V. Menezes and A.J. Bhattacharyya, J. Phys. Chem. B, 2010, 114, 5233
23 S. Das and A.J. Bhattacharyya, J. Phys. Chem. B, 2011, 115, 2148

24 S. Das, S.J. Prathapa, P.V. Menezes, T.N. Guru Row and A.J. Bhattacharyya, J. Phys. Chem. B, 2009, 113, 5025

25 Th. Bauer, M. Köhler, P. Lunkenheimer, A. Loidl and C.A. Angell, J. Chem. Phys., 2010, 133, 144509

26 J.LI. Tamarit, I.B. Rietveld, M. Barrio and R. Céolin, J. Mol. Struct., 2014, 1078, 3

27 M. Götz, Th. Bauer, P. Lunkenheimer and A. Loidl, J. Chem. Phys., 2014, 140, 094504

28 J.M. Pringle, P.C. Howlett, D.R. MacFarlane and M. Forsyth, J. Mater. Chem., 2010, 20, 2056

29 J.M. Pringle, Phys. Chem. Chem. Phys., 2013, 15, 1339

30 F. Kremer and A. Schönhals, Broad Band Dielectric Spectroscopy (Springer, Berlin, 2003), chapters 3 and 4

31 D.V. Nickel, H. Bian, J. Zheng and D.M. Mittleman, J. Infrared Milli. Terahz Waves, 2014, 35, 770

32 C.A. Angell, J. Non-Cryst. Solids, 1988, 102, 205

33 C.A. Angell, J. Non-Cryst. Solids, 1991, 131, 13

34 M. Petrowsky and R. Frech, J. Phys. Chem. B, 2009, 113, 5996

35 R. Frech and M. Petrowsky, J. Phys. Chem. B, 2014, 118, 2422

36 R. Brand, P. Lunkenheimer and A. Loidl, J. Chem. Phys., 2002, 116, 10386

37 M.A. Ramos, S. Vieira, F.J. Bermejo, J. Dawidowski, H.E. Fischer, H. Schober, M.A. González, C.K. Loong and D.L. Price, Phys. Rev. Lett., 1997, 78, 82

38 S. Benkhof, A. Kudlik, T. Blochowicz and E. Rössler, J. Phys.: Condens. Matter, 1998, 10, 8155

39 M.A. Miller, M. Jiménez-Ruiz, F.J. Bermejo and N.O. Birge, Phys. Rev. B, 1998, 57, R13977

40 M. Jiménez-Ruiz, M.A. Gonzáles, F.J. Bermejo, M.A. Miller, N.O. Birge, I. Cendoya and A. Alegría, Phys. Rev. B, 1999, 59, 9155

41 R. Macovez, M. Zachariah, M. Romanini, P. Zygouri, D. Gournis and J.Ll. Tamarit, J. Phys. Chem. C, 2014, 118, 12170-12175

42 M. Zachariah, E. Mitsari, M. Romanini, P. Zygouri, D. Gournis, M. Barrio, J.LI. Tamarit and R. Macovez, J. Phys. Chem. C, 2015, 119, 685-694 